\title{
Cleaner Production Quality Regulation Strategy of Pharmaceutical with Collusive Behavior and Patient Feedback
}

\author{
Junmei Rong ${ }^{1,2}$ and Lilong Zhu $\mathbb{D}^{1}$ \\ ${ }^{1}$ School of Business, Shandong Normal University, Jinan, Shandong 250014, China \\ ${ }^{2}$ School of Economics and Management, Tongji University, Shanghai 200092, China \\ Correspondence should be addressed to Lilong Zhu; zhulilong2008@126.com
}

Received 14 May 2020; Revised 6 July 2020; Accepted 13 July 2020; Published 17 August 2020

Academic Editor: Dimitri Volchenkov

Copyright (c) 2020 Junmei Rong and Lilong Zhu. This is an open access article distributed under the Creative Commons Attribution License, which permits unrestricted use, distribution, and reproduction in any medium, provided the original work is properly cited.

\begin{abstract}
The quality of pharmaceuticals has always been a hot issue in the world, and it involves public health, economic development of countries, social stability, and national security. Cleaner production is a prerequisite for ensuring the quality of medicines. However, the various types of counterfeit pharmaceuticals and fake vaccines exposed in the recent years have revealed many problems, such as lacking government regulation, loose quality management of companies, illegal profit of medical service agencies, and failure of patient complaints. This paper's two innovations are as follows: first, it not only considers the collusion between pharmaceutical companies and medical service agencies, but also introduces patient feedback to study drug quality regulation strategies from a microperspective; second, this paper constructs a tripartite evolutionary game model involving cleaner production pharmaceutical companies, medical service agencies, and the governments to analyze the evolutionary stability using the Lyapunov first rule. The results of the research show that, first, improvement of patient complaint rates can effectively curb collusive behavior and promote the stable improvement of cleaner production drug quality; second, the governments must impose sufficient fines on pharmaceutical companies to avoid a stable strategic combination of collusion; third, enhancing patient feedback can speed up the evolution of the stable choice of legitimate strategies by pharmaceutical companies and the medical service agencies; finally, the government reducing the strict regulation costs can increase the strict regulation rate employed in the evolution process and slow down the pace of evolution to loose regulation. In addition, the simulation analysis was carried out using Matlab 2017b, which verified the validity of the model and proved the practical meaning of countermeasures and suggestions for improving government quality regulation.
\end{abstract}

\section{Introduction}

The quality of pharmaceuticals has always been a hot issue in the world, and it involves public health, economic development of countries, social stability, and national security. The implementation of cleaner production of pharmaceuticals not only ensures the quality and safety of pharmaceuticals, but also promotes the sustainable development of the environment [1]. However, according to data reported by the World Health Organization (WHO), the proportion of counterfeit drugs worldwide is approximately $5 \%$ to $10 \%$. In recent years, various types of drug safety and quality incidents have occurred frequently. In 2010, the US Food and Drug Administration issued safety warnings for two drugs produced by GlaxoSmithKline (GSK) more than once; one of these drugs caused an increased risk of worsening asthma, leading to an increased probability of hospitalization and even death of the patients. In 2017, the US pharmaceutical giant Johnson \& Johnson issued a statement saying that more than 40 batches of unqualified drugs produced by the subsidiary McNeil were recalled from 12 countries and regions such as the United States and Canada. On July 20, 2018, the "fake vaccine" incident was exposed in China, in which the Changchun Changsheng Company had illegally produced fake vaccines for four years. In 2019, many Japanese eye drops sold worldwide were ordered off the shelves by the Canadian Health Department due to safety suspicions. These incidents have fully exposed many 
problems such as inadequate drug quality regulation in many countries, lack of cleaner production quality management in pharmaceutical companies, and illegal profits of medical service agencies. In 2017, the statistical annual report of the China National Medical Product Administration showed that a total of 112,000 cases of counterfeit drugs were investigated, with a year-over-year growth rate of $15.7 \%$. The inferior and low-quality drugs continue to threaten public and health.

On the one hand, pharmaceutical companies face huge challenges, especially small- and medium-sized enterprises. Drugs have the characteristics of high research and development costs, strictly sterile production environment, long clinical trial periods, strict production process standards, and limited prices. These have brought huge challenges to operation of small- and medium-sized pharmaceutical companies. To reduce costs and shorten the test period, companies may use low-quality raw materials, change the inspection records, or even lower the cleaner production standards. These violations not only damage the quality of drugs, but also expand carbon emissions [2]. According to Chan and Daim [3], China's pharmaceutical companies have been facing problems related to staffing shortages and quality management. As drugs account for approximately half of the total health spending in China [4], it was undeniable that drugs are an important part of health care.

On the other hand, medical service agencies and pharmaceutical companies have a motivated interest in collusion. Different from general commodities, drugs have the property of trusted goods, and patients who do not have medical expertise need to rely on medical service agencies to select drugs. Therefore, there is a health information asymmetry between doctors and patients [5]. In the stages of new drug research and development, clinical trials, and adverse reaction reports, pharmaceutical companies need accurate data support from medical service agencies and recommendations from medical experts. In the 2013 GSK bribery case, bribes were paid by GSK to Chinese pharmaceutical associations, hospitals, and doctors to open up sales channels in the Chinese market. Driven by interests, pharmaceutical companies have the incentive to collude with medical service agencies, thereby reducing capital investment and still gaining more market share. Medical service agencies are also motivated to collaborate with pharmaceutical companies, tampering with clinical data and concealing adverse pharmaceutical reactions for the benefit of collusion. In addition, it is particularly noteworthy that the level of medical knowledge of patients is constantly improving and information barrier of medical service agencies is gradually broken, with the improvement of education and medical experience $[6,7]$.

Therefore, there are two innovations: first, this paper not only considers the collusion between pharmaceutical companies and medical service agencies, but also introduces patient feedback to study drug quality regulation strategies from a microperspective; second, this paper constructs a tripartite evolutionary game model involving cleaner production pharmaceutical companies, medical service agencies, and the governments to analyze the evolutionary stability using the Lyapunov first rule. The three issues discussed in this paper are as follows: How to establish a tripartite evolutionary game model for cleaner production quality regulation considering collusive behavior between medical service agencies and pharmaceutical companies? How do patient feedback and other factors affect the strategic choices of each gamer? How can effective countermeasures be adopted to avoid conspiracies to ensure the high quality of pharmaceuticals?

The rest of the paper is organized as follows. Section 2 is the related literatures. The model hypothesis and construction are given in Section 3. Then, Section 4 contains the evolutionary stability analysis of each player's strategy and the stability analysis of the equilibrium points for the whole replicator dynamic system. Section 5 presents the numerical simulation analysis in a three-dimensional space using the software Matlab 2017b. The conclusions are given in Section 6.

\section{Related Literatures}

This section reviews the literature from three directions: the factors affecting the cleaner production quality of pharmaceuticals, countermeasure to improve pharmaceutical quality regulation, and applicability of a tripartite evolutionary game method.

First, there are many factors affecting the quality of pharmaceuticals. Industrial responsibility plays an important role in drug safety [8]. Cleaner production quality is the core of drug safety. But, factors like the lower production management level [9], poor anticounterfeiting technology [10], parasitic behavior [11], and complexity of the pharmaceutical supply chain all aggravate the urgency of solving drug safety issues. The influence of medical service agencies and the medical knowledge levels of patients has attracted the attention of scholars. Bardwell et al. [12] suggested that pharmaceutical checking programming should be used by drug retailers. The medical service agencies' checking has a significant impact on quality results [13]. Doctors and pharmaceutical-related industries have close relationships [14]. And, pharmaceutical companies always tailor their message content to a doctor, in order to enter the hospital market [15]. In fact, doctors are responsible for the health of patients while also playing the role of drug brokers or retailers. Considering the impact of patient feedback, decreasing the information disadvantage of patients is the key to preventing deception by experts [16]. Therefore, the research on the impact of collusive behavior between pharmaceutical companies and medical service agencies on drug quality is lacking, and the research on the role of patient feedback is not sufficient.

Second, constructing an effective regulation system to eliminate counterfeit and inferior drugs has become a research highlight for scholars. In recent years, China's adverse drug reaction (ADR) monitoring system has functioned well, but it also needs to enhance risk control actions to improve drug safety [17]. Donelan et al. [18] analyzed the main factors of drug quality from the perspective of regulatory and cleaner production. The more severe penalties 
from the Food and Drug Administration (FDA) have a unique positive effect on quality importance [19]. According to $\mathrm{Yi}$ et al. [20], China's health care providers have grown heavily reliant on revenue from drugs; this reliance is not conducive to drug safety. In addition, relying solely on government regulatory strategies such as permits, inspections, and penalties is no longer appropriate. The sustainable pharmaceutical recycling network is constantly being built and improved [21]. Developing dynamic monitoring system has become a trend [22]. The government regulatory authority should pay attention to the establishment of a safety evaluation system and a compensation system for adverse drug reactions and focus on the monitoring of adverse drug reaction data [23]. Therefore, most of the current research on drug quality regulation is carried out from the macrolevel, and there is a lack of consideration of the impact of various stakeholder behaviors from the microlevel.

Third, it is reasonable to use evolutionary game theory to analyze the quality regulation strategy of pharmaceuticals. Evolutionary game theory is an effective method to study the dynamic changes in the strategies of bounded rational subjects in a long-term repeated game under asymmetric information [24]. Abram and Noray [25] constructed a twopopulation evolutionary game model to research the rate of detection of corrupt politicians. Of course, the tripartite evolutionary game theory also has a wide range of applications. For example, Jiang et al. [26] constructed a threeparty game model that was analyzed and simulated by adopting dynamics to obtain the regulation strategies for an online shopping problem; Luo et al. [27] constructed and researched a tripartite game model of health food safety risk evolution to provide a theoretical basis for effective market governance; Long et al. [28] constructed a tripartite game model involving the government, consumers, and companies to explore the evolutionary equilibrium and the main driving factors for environmental problems; Liu et al. [29] analyzed the stability of a coal mine safety monitoring system based on tripartite evolutionary game theory and proposed forward control schemes such as dynamic fines. Therefore, it is reasonable and innovative to use the threeparty evolutionary game theory to study the cleaner production quality regulation strategy of the pharmaceutical.

In summary, the existing literature is extremely lacking tripartite game analysis among the governments, cleaner production pharmaceutical companies, and medical service agencies, as well as the consideration of collusive behavior and patient feedback. Using Lyapunov's first rule can enrich the stability research of the three-party evolutionary game model.

\section{Model Hypothesis and Construction}

3.1. Model Hypothesis. In addition to cleaner producing high-quality drugs, pharmaceutical companies may supply low-quality drugs for medical service agencies. Low-quality drugs refer to inaccurate clinical data, inconsistent indications, poor efficacy, unqualified quality, and other situations. If a medical service agency adopts a collusive strategy, it will receive low-quality drugs for diagnosis. Collusion includes illegal and profit-seeking behaviors, such as accepting bribes from pharmaceutical companies, tampering with clinical data, concealing adverse reactions, and illegally expanding doses and indications. When the medical service agency adopts a noncollusive strategy, it will reject low-quality drugs and guarantee the quality of the drugs as well as the effect of treatment. At this time, patients will provide positive feedback (giving a banner and positive publicity). However, if collusion occurs, patients who are treated under collusive behavior will provide negative feedback (distributing negative speech or even medical disputes) due to poor efficacy or adverse reactions, and they will decide whether to complain to the government. The government regulatory authority performs the supervisory duties. In daily management systems, cleaner production pharmaceutical companies are clearly required to provide pharmaceutical information, such as production inspections and clinical data; medical service agencies are required to collect medical information, such as medical records. In addition, the government will choose to adopt strict or loose regulatory strategies to prevent the occurrence of drug quality problems. Therefore, the logical relationship of the game model is shown in Figure 1.

Because patients do not have professional medical knowledge, they are at a disadvantage in information, and their purchase decision greatly depends on the medical service agencies. Therefore, this paper selects the government regulatory authority, pharmaceutical companies, and medical service agencies as the main players. The game model not only considers the collusive behavior of pharmaceutical companies and medical service agencies, but also considers the impact of the patient feedback mechanism. Assume the following:

Hypothesis 1. The pharmaceutical companies are participant 1 , and their strategic space is $\alpha=\left(\alpha_{1}, \alpha_{2}\right)=$ (cleaner producing high-quality drugs with a probability of $x$, cleaner producing low-quality drugs with a probability of $1-x$ ). The medical service agencies are participant 2 , and their strategic space is $\beta=\left(\beta_{1}, \beta_{2}\right)=$ (collusion with a probability of $y$, noncollusion with a probability of $1-y$ ). The government regulatory authorities are participant 3 , and their strategic space is $\gamma=\left(\gamma_{1}, \gamma_{2}\right)=$ (strict regulation with a probability of $z$, loose regulation with a probability of $1-z)$. These three players represent three bounded rational groups.

Hypothesis 2. Pharmaceutical companies generate revenue $R_{p}$ by cleaner producing and selling drugs. The cost of cleaner producing high-quality drugs is $C_{\mathrm{ph}}$; the cost of cleaner producing low-quality drugs is $C_{\mathrm{pl}}$, $C_{\mathrm{ph}}>C_{\mathrm{pl}}$. When cleaner production pharmaceutical companies choose to supply low-quality drugs, they need to collude with medical services, i.e., choose a collusive strategy. In this situation, pharmaceutical companies will pay conspiracy costs to medical services, and collusion will provide additional benefits to pharmaceutical companies; we set the conspiracy cost to be $C_{\mathrm{pb}}$ and the additional benefits to be $W_{p}$. 


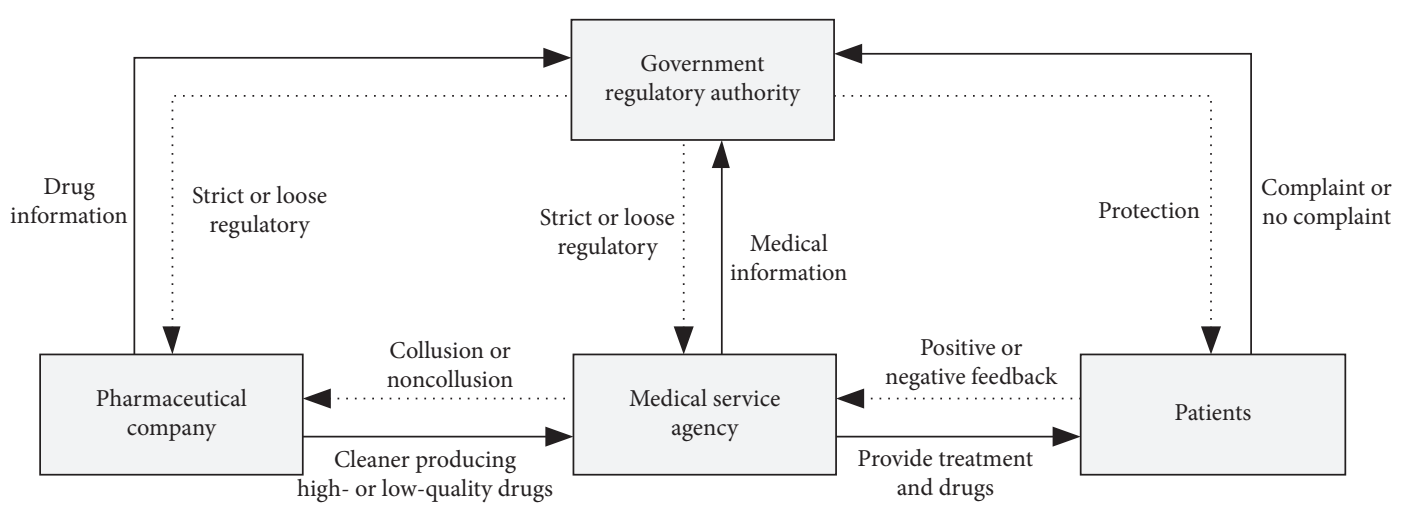

FIGURE 1: The logical relationship of the game model.

Hypothesis 3. The medical service agencies provide patients with medical treatment and drugs that generate income $R_{s}$. If the medical service agency colludes with the pharmaceutical company, low-quality drugs will be provided, and the additional benefit that the collusion brings to medical service agencies is $W_{\mathrm{sb}}$. If the medical service chooses a noncollusive strategy, it will not purchase low-quality drugs.

Hypothesis 4. If the patients are treated under a collusion, the patients' recovery is slow, the medical cost increases, and even an adverse reaction occurs. In this situation, the patients will provide negative feedback to the medical service agencies (distributing negative speech and medical trouble), which is not conducive to social stability and economic development. Thus, the reputation loss suffered by the medical service agencies is $\Psi_{s}$. In addition, the collusive strategy increases the operational cost of the medical service agencies, and the operating cost is $C_{s}$. The social cost of the governments to rectify medical development and maintain social stability is $\Psi_{g}$. If the patients are treated with highquality drugs in a noncollusion situation, then the patients recover quickly and will provide positive feedback to the medical service agencies (giving a commendation and positive publicity), which is conducive to the development of medical care. In this situation, the medical service agencies obtain the reputation value $\Upsilon_{s}$, and the governments receive the social benefits $\Upsilon_{g}$.

Hypothesis 5. When collusion occurs and the governments' regulation is loose, the patients have a likelihood of $\xi(0<\xi<1)$ to identify collusive behavior or drug quality problems and complain to the governments (i.e., $\xi$ is the complaint rate of patients). At this time, the government regulatory authorities are subject to administrative penalties $T_{g}$ (dismissal, responsibility, and transfer to prosecution) by superiors due to lack of regulation. The government regulatory authorities adopt strict regulation after receiving complaints from patients, and the cost of strict regulation is $C_{g}$. If pharmaceutical companies provide low-quality drugs that are strictly regulated, they will be fined $F_{p}$. If medical service agencies are found involved in the collusion, they will be fined $F_{s}$.

3.2. Model Construction. Based on the above assumptions, the hybrid strategy game matrix among the government regulatory authorities, the cleaner production pharmaceutical companies, and the medical service agencies is shown in Table 1 that considers collusive behavior and patient feedback.

\section{Model Analysis}

4.1. Evolutionary Stability Analysis of Pharmaceutical Companies' Strategies. According to the mixed strategy game matrix, cleaner production pharmaceutical companies' expected return of producing high-quality drugs is $E_{11}$, the expected return of producing low-quality drugs is $E_{12}$, and $\overline{E_{1}}$ is the average expected return. These expectation functions are shown in the following equation group:

$$
\left\{\begin{array}{l}
E_{11}=z\left[R_{p}-C_{\mathrm{ph}}\right]+(1-z)\left[R_{p}-C_{\mathrm{ph}}\right] \\
E_{12}=z\left[y\left(R_{p}-C_{\mathrm{pl}}-C_{\mathrm{pb}}+W_{p}-F_{p}\right)+(1-y)\left(-C_{\mathrm{pl}}-F_{p}\right)\right]+(1-z)\left[y\left(R_{p}-C_{\mathrm{pl}}-C_{\mathrm{pb}}+W_{p}-\xi F_{p}\right)+(1-y)\left(-C_{\mathrm{pl}}\right)\right] \\
\overline{E_{1}}=x E_{11}+(1-x) E_{12} .
\end{array}\right.
$$


TABLE 1: The hybrid strategy game matrix for the tripartite evolution game model.

\begin{tabular}{|c|c|c|c|c|}
\hline & & \multirow{2}{*}{$\begin{array}{l}\text { Medical service } \\
\text { agencies }\end{array}$} & \multicolumn{2}{|c|}{ Government regulatory authorities } \\
\hline & & & Strict regulation $z$ & Loose regulation $1-z$ \\
\hline \multirow{4}{*}{$\begin{array}{l}\text { Pharmaceutical } \\
\text { companies }\end{array}$} & $\begin{array}{l}\text { Cleaner } \\
\text { producing high- } \\
\text { quality drugs } x\end{array}$ & Collusion $y$ & $R_{p}-C_{\mathrm{ph}}, R_{s}-C_{s}+\Upsilon_{s}, \Upsilon_{g}-C_{g}$ & $R_{p}-C_{\mathrm{ph}}, R_{s}-C_{s}+\Upsilon_{s}, \Upsilon_{g}$ \\
\hline & \multirow{3}{*}{$\begin{array}{c}\text { Cleaner } \\
\text { producing } \\
\text { low-quality drugs } \\
1-x\end{array}$} & $\begin{array}{c}\text { Noncollusion } \\
1-y\end{array}$ & $R_{p}-C_{\mathrm{ph}}, R_{s}+\Upsilon_{s}, \Upsilon_{g}-C_{g}$ & $R_{p}-C_{\mathrm{ph}}, R_{s}+\Upsilon_{s}, \Upsilon_{g}$ \\
\hline & & Collusion $y$ & $\begin{array}{c}R_{p}-C_{\mathrm{pl}}-C_{\mathrm{pb}}+W_{p}-F_{p} \\
R_{s}+W_{\mathrm{sb}}-C_{s}-\Psi_{s}-F_{s} \\
F_{p}+F_{s}-C_{g}-\Psi_{g}\end{array}$ & $\begin{array}{c}R_{p}-C_{\mathrm{pl}}-C_{\mathrm{pb}}+W_{p}-\xi F_{p} \\
R_{s}+W_{s b}-C_{s}-\Psi_{s}-\xi F_{s} \\
\xi\left(F_{p}+F_{s}-C_{g}-T_{g}\right)-\Psi_{g}\end{array}$ \\
\hline & & $\begin{array}{l}\text { Noncollusion } \\
1-y\end{array}$ & $-C_{\mathrm{pl}}-F_{p}, R_{s}+\Upsilon_{s}, \Upsilon_{g}+F_{p}-C_{g}$ & $-C_{\mathrm{pl}}, R_{s}+\Upsilon_{s}, \Upsilon_{g}$ \\
\hline
\end{tabular}

The replicator dynamic equation and its first derivative are

$$
\begin{aligned}
F(x)= & \frac{\mathrm{d} x}{\mathrm{~d} t}=x\left(E_{11}-\overline{E_{1}}\right) \\
= & x(x-1)\left[C_{\mathrm{ph}}-C_{\mathrm{pl}}-R_{p}+y\left(R_{p}-C_{\mathrm{pb}}+W_{p}\right.\right. \\
& \left.\left.-\xi F_{p}\right)-z F_{p}+y z \xi F_{p}\right], \\
\frac{d(F(x))}{\mathrm{d} x}= & (2 x-1)\left[C_{\mathrm{ph}}-C_{\mathrm{pl}}-R_{p}+y\left(R_{p}-C_{\mathrm{pb}}+W_{p}\right.\right. \\
& \left.\left.-\xi F_{p}\right)-z F_{p}+y z \xi F_{p}\right] .
\end{aligned}
$$

Set

$G(z)=C_{\mathrm{ph}}-C_{\mathrm{pl}}-R_{p}+y\left(R_{p}-C_{\mathrm{pb}}+W_{p}-\xi F_{p}\right)-z F_{p}+y z \xi F_{p}$.

If $C_{\mathrm{ph}}-C_{\mathrm{pl}}-C_{\mathrm{pb}}+W_{p}-\xi F_{p}<0$, then $G(z)<0$; the solutions for $F(x)=0$ are $x=0$ and $x=1$. According to the fundamental theorem of stability of differential equations,
$\mathrm{d}(F(x)) /\left.\mathrm{d} x\right|_{x=1}<0$ and $\mathrm{d}(F(x)) /\left.\mathrm{d} x\right|_{x=0}>0$, so $x=1$ is the ESS (evolutionary stable strategy).

If $C_{\mathrm{ph}}-C_{\mathrm{pl}}-C_{\mathrm{pb}}+W_{p}-\xi F_{p} \geq 0$, then the solutions for $F(x)=0$ are $x=0, x=1$, and $G(z)=0$ (the solution is $z=$ $\left[C_{\mathrm{ph}}-C_{\mathrm{pl}}-R_{p}+y\left(R_{p}-C_{\mathrm{pb}}+W_{p}-\xi F_{p}\right)\right] /\left[(1-y \xi) F_{p}\right]=$ $\left.z^{*}\right)$. According to the stability theorem of differential equations, we can obtain the following:

(1) When $z=z^{*}, G(z)=0$ and $F(x) \equiv 0$; thus, there is no ESS

(2) When $z<z^{*}, G(z)>0$ and $\mathrm{d}(F(x)) /\left.\mathrm{d} x\right|_{x=0}<0$; thus, $x=0$ is the ESS

(3) When $z>z^{*}, G(z)<0$ and $\mathrm{d}(F(x)) /\left.\mathrm{d} x\right|_{x=1}<0$; thus, $x=1$ is the ESS

The evolutionary trend of their strategy is shown in Figure 2.

From Figure 2, the probability that pharmaceutical companies choose to cleaner produce low-quality drugs equals the volume of $A_{1}$, recorded as $V_{A_{1}}$, and the probability of producing high-quality drugs equals the volume of $A_{2}$, recorded as $V_{A_{2}}$. Assuming $a=R_{p}-C_{\mathrm{ph}}+C_{\mathrm{pl}}$ and $b=R_{p}-C_{\mathrm{pb}}+W_{p}-\xi F_{p}$, the following is calculated:

$$
\begin{aligned}
V_{A_{1}} & =\int_{0}^{1} \int_{a / b}^{1} \frac{-a+y b}{F_{p}-y \xi F_{p}} \mathrm{~d} y \mathrm{~d} x=\frac{[b-\xi(b-a)+(b-\xi a) \ln (b-a \xi) /(b-b \xi))]}{\xi^{2} F_{p}} \\
V_{A_{2}} & =1-\int_{0}^{1} \int_{a / b}^{1} \frac{a+y b}{F_{p}-y \xi F_{p}} \mathrm{~d} y \mathrm{~d} x=\frac{\left.\left[\xi^{2} F_{p}-b+\xi(b-a)-(b-\xi a) \ln (b-a \xi) /(b-b \xi)\right)\right]}{\xi^{2} F_{p}} .
\end{aligned}
$$

Proposition 1. With the increase in patient complaint rates, cleaner production pharmaceutical companies will reduce the availability of low-quality drugs, and pharmaceutical companies will provide high-quality drugs steadily until the complaint rate increases to $\xi>\left(C_{p h}-C_{p l}-C_{p b}+W_{p}\right) / F_{p}$. 


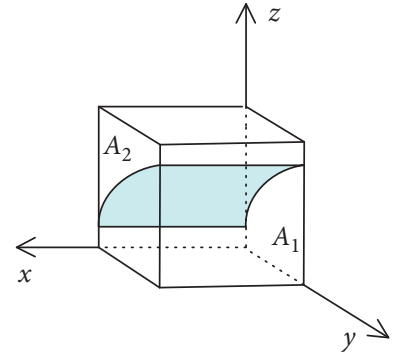

(a)

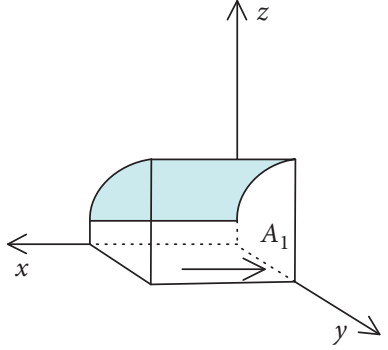

(b)

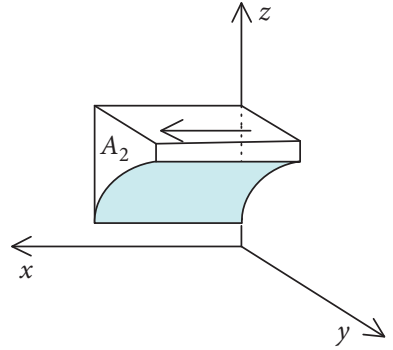

(c)

Figure 2: The evolutionary trend of pharmaceutical companies' strategy. (a) $z=z^{*}$. (b) $z<z^{*}$. (c) $z>z^{*}$.

Proof.

$$
\begin{aligned}
& \frac{\partial V_{A_{1}}}{\partial \xi}=\frac{\left[-\left(F_{p} \xi+2 b-a \xi\right) \ln ((b-a \xi) /(b-b \xi))-F_{p} \xi(1-\xi+(a \xi / b))-(b /(1-\xi))\left(2-4 \xi+\xi^{2}\right)-\left(a \xi^{2} /(1-\xi)\right)-2 \xi a\right]}{\xi^{3} F_{p}}, \quad \xi \in[0,1] \\
& \quad \text { when } \xi<\frac{\left(C_{\mathrm{ph}}-C_{\mathrm{pl}}-C_{\mathrm{pb}}+W_{p}\right)}{F_{p}}, \\
& \quad C_{\mathrm{ph}}-C_{\mathrm{pl}}-C_{\mathrm{pb}}+W_{p}-\xi F_{p}<0 \\
& \quad \frac{\partial V_{A_{1}}}{\partial \xi}<0
\end{aligned}
$$

which means that increasing the patient complaint rate will cause pharmaceutical companies to reduce the cleaner production of low-quality drugs. When the complaint rate rises to $\xi>\left(C_{\mathrm{ph}}-C_{\mathrm{pl}}-C_{\mathrm{pb}}+W_{p}\right) / F_{p}$, i.e., $C_{\mathrm{ph}}-C_{\mathrm{pl}}-C_{\mathrm{pb}}+$ $W_{p}-\xi F_{p}<0, x=1$ will be the ESS.

Proposition 1 indicates that while paying attention to green ecological protection, the governments should widely provide public education on medical knowledge. Many behaviors of patients can effectively reduce the incidence of pharmaceutical companies providing low-quality drugs, such as their ability to determine the quality of drugs and their strong sense of rights. And, governments should ensure that patients have a way to complain, so that patients can actively participate in the drug quality supervision, to jointly create a high-quality drug market.

Proposition 2. Increasing the amount of strict regulation by the governments will help ensure pharmaceutical companies to cleaner produce high-quality drugs, and the fine imposed by the government on pharmaceutical companies must be no less than $\left(W_{p}-C_{p b}+C_{p h}-C_{p l}\right) /(z+\xi-z \xi)$; otherwise, a large number of low-quality drugs will be added to the drug market.

Proof. Because $\partial G(z) / \partial z<0, G\left(z<z^{*}\right)>0, G\left(z=z^{*}\right)=0$, and $G\left(z>z^{*}\right)<0$ and according to the evolutionary stability analysis of pharmaceutical companies' strategies, as $z$ increases from $z<z^{*}$ to $z>z^{*}$, the evolutionary stable strategy evolves from $x=0$ to $x=1$. In addition, let $y^{*}=\left[R_{p}-C_{\mathrm{ph}}+C_{\mathrm{pl}}+z F_{p}\right] /\left[R_{p}-C_{\mathrm{pb}}+W_{p}-\xi F_{p}+z \xi F_{p}\right]$ and $G\left(y=y^{*}\right)=0$. When $F_{p}>\left(R_{p}-C_{\mathrm{pb}}+W_{p}\right) / \xi(1-z)$, $\partial G(y) / \partial y<0$, and $y>y^{*}, G(y)<0$, so $x=1$ is the ESS. When $\left(W_{p}-C_{\mathrm{pb}}+C_{\mathrm{ph}}-C_{\mathrm{pl}}\right) / \quad(z+\xi-z \xi)<F_{p}<\left(R_{p}-\right.$ $\left.C_{\mathrm{pb}}+W_{p}\right) / \xi(1-z), \partial G(y) / \partial y>0$, and $y<y^{*}, G(y)<0$, so $x=1$ is the ESS. When $F_{p}<\left(W_{p}-C_{\mathrm{pb}}+C_{\mathrm{ph}}-C_{\mathrm{pl}}\right) /$ $(z+\xi-z \xi)$, as $y$ increases from $y<y^{*}$ to $y>y^{*}$, the ESS will change from $x=1$ to $x=0$.

Proposition 2 indicates that the government regulatory authorities should set a reasonable fine, greater than a specific threshold. And, the information construction of the pharmaceutical industry is indispensable. The more the information on cleaner production of drugs collected by the government regulatory authorities, the more the effective punishment mechanisms can be designed through reasonable analysis to create a high-quality drug market and fully ensure the safety of the drug. In other words, avoiding excessive punishment can help the development of cleaner production of drugs, avoiding drug shortage, and curbing the rise of its prices.

Corollary 1. Increasing the percentage of the collusive cost by $1 \%$ or decreasing the percentage of the additional benefit of collusion by $1 \%$ will cause providing high-quality drugs to increase by $k \%(k=[\ln ((b-a \xi) /(b-b \xi))+(a \xi / b)+$ $\left.(1-\xi)] / \xi^{2} F_{p}\right)$. In addition, increasing the amount of fines 
imposed by the governments will increase the probability of pharmaceutical companies producing high-quality drugs, and the growth rate is more than $k \%$.
Proof. Find the first-order partial derivative of each element for $V_{A_{2}}$ :

$$
\begin{aligned}
\frac{\partial V_{A_{2}}}{\partial C_{\mathrm{pb}}}= & \frac{\partial V_{A_{1}}}{\partial b}=\frac{[\ln ((b-a \xi) /(b-b \xi))+(a \xi / b)+(1-\xi)]}{\xi^{2} F_{p}} ; \\
\frac{\partial V_{A_{2}}}{\partial W_{p}}=-\frac{\partial V_{A_{2}}}{\partial b}=-\frac{[\ln ((b-a \xi) /(b-b \xi))+(a \xi / b)+(1-\xi)]}{\xi^{2} F_{p}} & \\
\frac{\partial V_{A_{2}}}{\partial F_{p}}= & \frac{[\ln ((b-a \xi) /(b-b \xi))+(1-\xi)+(a \xi / b)]}{\xi^{2} F_{p}} \\
& +\frac{[(b-a \xi) \ln ((b-a \xi) /(b-b \xi))+b(1-\xi)+a]}{\xi^{2} F_{p}^{2}}
\end{aligned}
$$

Obviously, $\quad \partial V_{A_{2}} / \partial C_{\mathrm{pb}}>0, \quad \partial V_{A_{2}} / \partial W_{p}<0$, $\partial V_{A_{2}} / \partial C_{\mathrm{pb}}=-\partial V_{A_{2}} / \partial W_{p}$, and $\partial V_{A_{2}} / \partial F_{p}>\partial V_{A_{2}} / \partial C_{\mathrm{pb}}>0$. Therefore, $V_{A_{2}}$ is an increasing function for $F_{p}$ and $C_{\mathrm{pb}}$ and it is a decreasing function for $W_{p}$. Their first-order partial derivatives are exactly their proportion of mutual influence.

Corollary 1 indicates that raising the costs of collusion to the pharmaceutical companies can promote cleaner production of high-quality medicines; reducing their additional benefit of collusion can also promote high-quality drugs. The governments can focus on cracking down on the distribution of inferior drugs through the introduction of relevant laws and regulations and strengthen public opinion pressure on pharmaceutical companies and medical service agencies. In addition, severe penalties and high fines imposed by the governments are effective measures to improve the quality of medicines.

4.2. Evolutionary Stability Analysis of Medical Service Agencies' Strategies. The expected return of the collusion strategy adopted by medical service agencies is $E_{21}$, the expected return of the noncollusion strategy is $E_{22}$, and $\overline{E_{2}}$ is the average expected return:

$$
\left\{\begin{array}{l}
E_{21}=x\left[R_{s}-C_{s}+\Upsilon_{s}\right]+(1-x)\left[R_{s}+W_{\mathrm{sb}}-C_{s}-\Psi_{s}-z F_{s}-(1-z) \xi F_{s}\right] \\
E_{22}=x\left[R_{s}+\Upsilon_{s}\right]+(1-x)\left[R_{s}+\Upsilon_{s}\right] \\
\overline{E_{2}}=y E_{21}+(1-y) E_{22} .
\end{array}\right.
$$

From the equation group (7), the replicator dynamic equation and its first derivative are

$$
\begin{aligned}
F(y)= & \frac{\mathrm{d} y}{\mathrm{~d} t}=y\left(E_{21}-\overline{E_{2}}\right) \\
= & y(y-1)\left[C_{s}+(1-x)\left(\Upsilon_{s}-W_{\mathrm{sb}}\right.\right. \\
& \left.\left.+\Psi_{s}+z F_{s}+(1-z) \xi F_{s}\right)\right], \\
\frac{\mathrm{d}(F(y))}{\mathrm{d} y}= & (2 y-1)\left[C_{s}+(1-x)\left(\Upsilon_{s}-W_{\mathrm{sb}}\right.\right. \\
& \left.\left.+\Psi_{s}+z F_{s}+(1-z) \xi F_{s}\right)\right] .
\end{aligned}
$$

Set

$$
J(z)=C_{s}+(1-x)\left(\Upsilon_{s}-W_{\mathrm{sb}}+\Psi_{s}+z F_{s}+(1-z) \xi F_{s}\right) .
$$

The solutions of $F(y)=0$ are $y=0, y=1$, and $J(z)=0$. And, the solution of $J(z)=0$ is $z=z^{* *}=\left[W_{\mathrm{sb}}-\Upsilon_{s}-\Psi_{s}-\xi F_{s}-C_{s} /(1-x)\right] /\left[(1-\xi) F_{s}\right]$, $z^{* *} \in(0,1)$. According to the stability theorem of differential equations, we can obtain the following:

(1) When $z=z^{* *}, F(y) \equiv 0$; thus, there is no ESS for medical service agencies

(2) When $z>z^{* *}, \mathrm{~d}(F(y)) /\left.\mathrm{d} y\right|_{y=0}<0$; thus, $y=0$ is the ESS

(3) When $z<z^{* *}, \mathrm{~d}(F(y)) /\left.\mathrm{d} y\right|_{y=1}<0$; thus, $y=1$ is the ESS

The evolutionary trend of medical service agencies' strategy is shown in Figure 3.

According to Figure 3, the probability of noncollusion chosen by the medical service agencies is equal to the volume $V_{B_{1}}$ of $B_{1}$; the probability of choosing collusion is equal to 


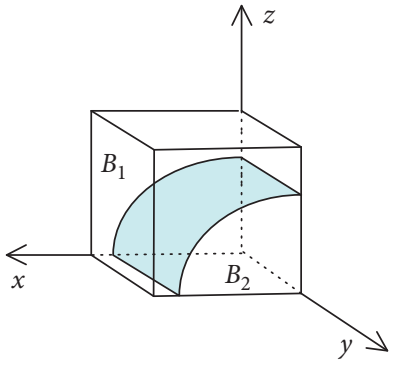

(a)

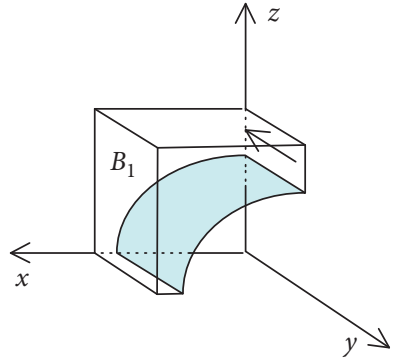

(b)

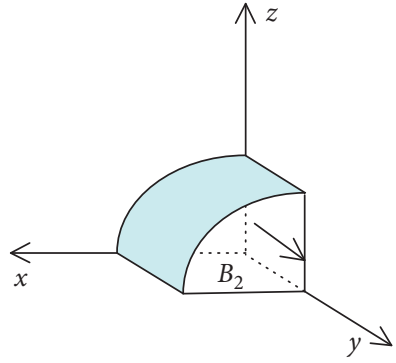

(c)

Figure 3: The evolutionary trend of medical service agencies' strategy. (a) $z=z^{*}$. (b) $z>z^{*}$. (c) $z<z^{*}$.

the volume $V_{B_{2}}$ of $B_{2}$. Let $n=W_{\mathrm{sb}}-\Upsilon_{s}-\Psi_{s}-\xi F_{s}$, and the calculation results are

$$
\begin{aligned}
V_{B_{1}} & =1-\int_{0}^{1} \int_{0}^{C_{s} / n_{s}} \frac{\left(n-C_{s}\right) /(1-x)}{(1-\xi) F_{s}} \mathrm{~d} x \mathrm{~d} y \\
& =1-\frac{C_{s}}{F_{s}(1-\xi)}\left[1+\ln \left(1-\frac{C_{s}}{n}\right)\right], \\
V_{B_{2}} & =\frac{C_{s}}{F_{s}(1-\xi)}\left[1+\ln \left(1-\frac{C_{s}}{n}\right)\right] .
\end{aligned}
$$

Proposition 3. If the benefits of the collusion of the medical service agencies are greater than $\Upsilon_{s}+\Psi_{s}+\xi F_{s}+z(1-\xi) F_{s}$, the reduced probability of cleaner producing high-quality drugs by the pharmaceutical companies will increase the probability of the medical service agencies adopting a collusion strategy.

Proof. If $\quad W_{\mathrm{sb}}<\Upsilon_{s}+\Psi_{s}+\xi F_{s}+z(1-\xi) F_{s}$, then $\partial J(z) / \partial x<0, J(z)>0$, and $\mathrm{d}(F(y)) /\left.\mathrm{d} y\right|_{y=0}<0$; therefore, noncollusion (i.e., $y=0$ ) is the evolutionary stable strategy. If $W_{\mathrm{sb}}>\Upsilon_{s}+\Psi_{s}+\xi F_{s}+z(1-\xi) F_{s}$, then $\partial J(z) / \partial x>0$. Obviously, with $x$ decreasing from $x>x^{* *}$ to $x<x^{* *}$, the ESS will change from $y=0$ to $y=1$.

Proposition 3 indicates that if the benefits of the collusion given by the pharmaceutical companies to the medical service agencies are lower than the opportunity losses of the medical service agencies, the medical service agencies will adopt a noncollusion strategy. Otherwise, if it is not possible to ensure that cleaner production pharmaceutical companies provide high-quality drugs, the government should be cautious in reducing the amount of strict regulation and should strengthen the regulation of pharmaceuticals whose benefits may be too high in collusion to prevent collusion between medical agencies and pharmaceutical companies.

Corollary 2. Positive and negative patient feedback and patient complaints are all conducive to standardizing the behavior of medical service agencies, and the rate of patient complaint has a greater impact. Increasing the rate of patient complaint is more helpful for medical service agencies to refuse collusion.
Proof. Based on $V_{B_{1}}$, an increase in $\Upsilon_{s}$ or $\Psi_{s}$ or $\xi$ will all cause $V_{B_{1}}$ to increase:

$$
\begin{aligned}
\frac{\partial V_{B_{1}}}{\partial Y_{s}}= & \frac{\partial V_{B_{1}}}{\partial \Psi_{s}}=\frac{C_{s}^{2}}{(1-\xi) F_{s}\left(n^{2}-C_{s} n\right)}>0, \\
\frac{\partial V_{B_{1}}}{\partial \xi}= & \frac{C_{s}^{2}}{(1-\xi)\left(n^{2}-C_{s} n\right)} \\
& -\frac{C_{s}}{(1-\xi)^{2} F_{s}}\left[1+\ln \left(1-\frac{C_{s}}{n}\right)\right]>0,
\end{aligned}
$$

and $\partial V_{B_{1}} / \partial \xi-\partial V_{B_{1}} / \partial \Upsilon_{s}>0$.

Corollary 2 indicates that patients actively learn basic medical knowledge and effectively defend their rights through complaints, which can improve the behavioral norms of medical service agencies and keep low-quality drugs off the market. In addition, the influence of patient feedback has an important impact on medical service agencies. In the electronic information age, patients should take the initiative to use the convenience and influence of the media to enhance their feedback, in order to eliminate the medical service agencies that adopt conspiracy behaviors.

Corollary 3. The decrease in the additional income and the increase in the reputation loss have the same effect on the collusive behavior of medical service agencies, but their effect is less than that of the government increasing the fine.

Proof.

$$
\begin{aligned}
\frac{\partial V_{B_{1}}}{\partial F_{s}} & =\frac{1+\ln \left(\left(1-C_{s}\right) / n\right)}{(1-\xi) F_{s}^{2}}+\frac{C_{s}^{2} \xi}{(1-\xi) F_{s}\left(n^{2}-C_{s} n\right)}>0 \\
\frac{\partial V_{B_{1}}}{\partial W_{s b}} & =-\frac{C_{s}^{2}}{(1-\xi) F_{s}\left(n^{2}-C_{s} n\right)}<0, \\
\text { so, } \frac{\partial V_{B_{1}}}{\partial \Psi_{s}} & =-\frac{\partial V_{B_{1}}}{\partial W_{s b}}, \\
\frac{\partial V_{B_{1}}}{\partial W_{s b}} & <-\frac{\partial V_{B_{1}}}{\partial F_{s}} .
\end{aligned}
$$


Therefore, $V_{B_{1}}$ is an increasing function for $F_{s}$ and a decreasing function for $W_{\mathrm{sb}}$, the decrease in $W_{\mathrm{sb}}$ and the increase in $\Psi_{s}$ have the same effect on $V_{B_{1}}$, and the increase in $F_{s}$ has a more significant effect.

Corollary 3 indicates that the governments can effectively increase the noncollusion tendency of medical service agencies by increasing the fines imposed on medical service agencies. In addition, the governments should pay close attention to high-profit medicines because the higher the conspiracy income of medical service agencies, the greater the tendency of collusion.

4.3. Evolutionary Stability Analysis of Governments' Strategies. The expected return $E_{31}$ of the government regulatory authorities choosing strict regulation, the expected return $E_{32}$ of loose regulation, and the average expected return $\overline{E_{3}}$ are as follows:

$$
\left\{\begin{array}{l}
E_{31}=x\left[\Upsilon_{g}-C_{g}\right]+(1-x)\left[y\left(F_{p}+F_{s}-C_{g}-\Psi_{g}\right)+(1-y)\left(\Upsilon_{g}+F_{p}-C_{g}\right)\right] \\
E_{32}=x \Upsilon_{g}+(1-x)\left[y \xi\left(F_{p}+F_{s}-C_{g}-T_{g}\right)-y \Psi_{g}+(1-y) \Upsilon_{g}\right] \\
\overline{E_{3}}=z E_{31}+(1-z) E_{32}
\end{array}\right.
$$

From equation (13), the replicator dynamic equation of the government regulatory authorities' strategy selection and its first derivative are

$$
\begin{aligned}
F(z)= & \frac{\mathrm{d} z}{\mathrm{~d} t}=z\left(E_{31}-\overline{E_{3}}\right) \\
= & z(z-1)\left[C_{g}-(1-x) y F_{s}+(1-x)\right. \\
& \left.y \xi\left(F_{p}+F_{s}-C_{g}-T_{g}\right)-(1-x) F_{p}\right], \\
\frac{\mathrm{d}(F(z))}{\mathrm{d} z}= & (2 z-1)\left[C_{g}-(1-x) y F_{s}+(1-x)\right. \\
& \left.y \xi\left(F_{p}+F_{s}-C_{g}-T_{g}\right)-(1-x) F_{p}\right] .
\end{aligned}
$$

Assume

$$
\begin{aligned}
H(x)= & C_{g}-(1-x) y F_{s}+(1-x) \\
& y \xi\left(F_{p}+F_{s}-C_{g}-T_{g}\right)-(1-x) F_{p} .
\end{aligned}
$$

Solutions with $F(z)=0$ include $z=0, z=1$, and $H(x)=0$ (calculated $x=x^{*}=1-C_{g} / y F_{s}+F_{p}+y \xi\left(T_{g}+\right.$ $\left.\left.C_{g}-F_{p}-F_{s}\right)\right)$. According to the stability theorem of differential equations, the results are as the following:

(1) When $x=x^{*}$, there is no ESS for the government regulatory authority

(2) When $x<x^{*}, z=1$ is the ESS

(3) When $x>x^{*}, z=0$ is the ESS

The evolutionary trend of government regulatory authorities' strategy is shown in Figure 4.

According to Figure 4, the probability of the government regulatory authorities choosing the strict regulation strategy is equal to the volume $V_{C_{2}}$ of $C_{2}$; the probability of choosing the loose regulation strategy is equal to the volume $V_{C_{1}}$ of $C_{1}$. Let $m=F_{s}+\xi\left(T_{g}+C_{g}-F_{s}-F_{p}\right)$, and the calculation results are

$$
\begin{aligned}
V_{C_{2}} & =\int_{0}^{1} \int_{0}^{1} 1-\frac{C_{g}}{y F_{s}+F_{p}+y \xi\left(T_{g}+C_{g}-F_{p}-F_{s}\right)} \mathrm{d} y \mathrm{~d} z \\
& =1-\frac{C_{g} \ln \left((1+m) / F_{p}\right)}{m} ; \\
V_{C_{1}} & =1-\int_{0}^{1} \int_{0}^{1} 1-\frac{C_{g}}{y F_{s}+F_{p}+y \xi\left(T_{g}+C_{g}-F_{p}-F_{s}\right)} \mathrm{d} y \mathrm{~d} z \\
& =\frac{C_{g} \ln \left((1+m) / F_{p}\right)}{m} .
\end{aligned}
$$

Proposition 4. Strengthening the quality of cleaner production by pharmaceutical companies will reduce the strict regulatory rate of government regulatory authorities, and the increased probability of medical service agencies choosing collusion will increase the rate of strict government regulatory.

Proof. Because $\quad \partial H(x) / \partial x>0$, so $H\left(x<x^{*}\right)<0$, $H\left(x=x^{*}\right)=0$, and $H\left(x>x^{*}\right)>0$. According to the evolutionary stability analysis of the strategic choice of the government regulatory authorities, as $x$ increases from $x<x^{*}$ to $x>x^{*}$, the evolutionary stable strategy evolves from $z=1$ to $z=0$. In addition, when $1-C_{g} / F_{p}<x<1$, as $y$ increases from $y<y^{* *}$ to $y>y^{* *}$, the evolutionary stable strategy of the government authorities evolves from $z=0$ to $z=1$.

Proposition 4 indicates that the governments have to use data analysis to clarify the probability of strategic choices for pharmaceutical companies and medical service agencies and then to develop reasonable regulatory strategies. The governments should not only increase the rate of strict regulation when the probability of cleaner producing highquality drugs is low, but also establish a reasonable strict regulation rate when the probability of cleaner producing high-quality drugs is high. High-frequency regulatory disturbances can maintain a stable strategy of providing highquality drugs. In addition, to address the collusive behavior, it is recommended to strengthen the evaluation of medical 


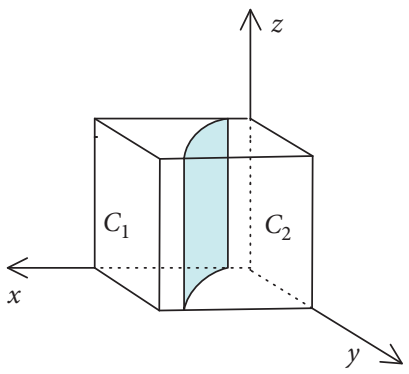

(a)

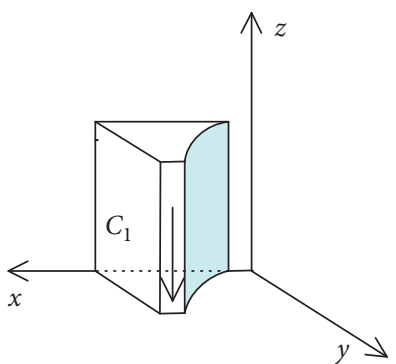

(b)

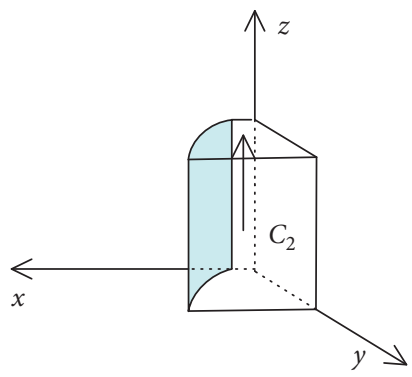

(c)

FIGURE 4: The evolutionary trend of government regulatory authorities' strategy. (a) $x=x^{*}$. (b) $x>x^{*}$. (c) $x<x^{*}$.

service agencies in the society and improve their moral standards.

Corollary 4. The increase in the rate of patient complaints will force government regulators to increase strict regulatory rates; the increase in administrative penalties by higher-level governments will also promote strict regulatory of drug quality.

Proof. $\partial V_{C_{2}} / \partial T_{g}=-C_{g} \xi\left[\left[1-F_{p} /\left(F_{p}+m\right)+\ln F_{p} /\left(F_{p}+m\right)\right] /\right.$ $m^{2}$ and $\partial V_{C_{2}} / \partial \xi=-C_{g}\left(T_{g}+C_{g}-F_{p}-F_{s}\right)\left[1-F_{p} /\left(F_{p}+m\right)+\right.$ $\left.\ln F_{p} /\left(F_{p}+m\right)\right] / m^{2}$. Obviously, $1-F_{p} /\left(F_{p}+m\right)+\ln F_{p} /$ $\left(F_{p}+m\right)<0, \partial V_{C_{2}} / \partial T_{g}>0$, and $\partial V_{C_{2}} / \partial \xi>0$; therefore, increasing $T_{g}$ and $\xi$ can increase the rate of strict regulatory $V_{\mathrm{C}_{2}}$.

Corollary 4 indicates that both the patient complaints and the penalties from higher-level governments could help the governments to strengthen the responsibility for drug safety and strict quality regulation to prevent the lack of regulation.

Corollary 5. With the reduction in strict regulation costs and the increase in the fines imposed on pharmaceutical companies and medical service agencies, the government regulatory authorities will increase the rate of strict regulation.

Proof. $V_{\mathrm{C}_{2}}$ is a decreasing function for $C_{g}$ and an increasing function for $F_{p}$ and $F_{s}$ because

$$
\begin{aligned}
& \frac{\partial V_{C_{2}}}{\partial C_{g}}=-\frac{\left[\left(m-\xi C_{g}\right) \ln \left(F_{p}+m\right) / F_{p}\right]}{m^{2}}<0 ; \\
& \frac{\partial V_{C_{2}}}{\partial F_{p}}=\frac{C_{g}\left(\xi+m / F_{p}\right)}{\left(F_{p}+m\right)}-\frac{\left[\xi C_{g} \ln \left(F_{p}+m\right) / F_{p}\right]}{m^{2}}>0 ; \\
& \frac{\partial V_{C_{2}}}{\partial F_{s}}=-\frac{C_{g}(1-\xi)\left[1-F_{p} /\left(F_{p}+m\right)+\ln F_{p} /\left(F_{p}+m\right)\right]}{m^{2}}>0 .
\end{aligned}
$$

Corollary 5 indicates that government regulators can improve the rate of strict regulation employed by adopting low-cost new technologies or innovative regulatory means. In addition, fines not only have a binding effect on pharmaceutical companies and the government regulatory authorities but also promote the use of the strict regulation strategy. The governments should increase fines imposed on illegal pharmaceutical companies and medical service agencies, which can effectively enhance strict regulation to ensure the cleaner production of high-quality medicines.

4.4. Analysis of Stability Equilibrium Points of Replicator Dynamic System. In the replicator dynamic system of the three-party game, the strategy selection of the participants starts from an initial point in any small field and evolves with stability to a certain equilibrium point and is robust to small disturbances; according to the stability concept of Lyapunov, this equilibrium point is a progressive ESS. According to the replicator dynamic equations, the Jacobian matrix of the dynamic system is

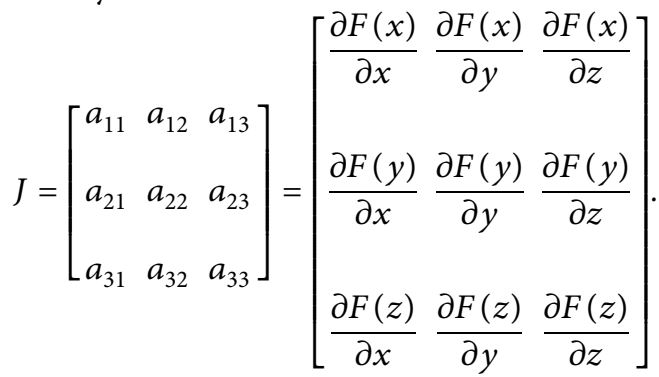

According to the Lyapunov first rule, if all the eigenvalues of the Jacobian matrix are negative, the equilibrium point is asymptotically stable; if the eigenvalues of the Jacobian matrix have at least one positive number, the equilibrium point is an unstable point; if the Jacobian matrix is divided by zero eigenvalues and the other negative eigenvalues, the equilibrium point is in a critical state, and the stability cannot be determined by the eigenvalue symbol. This method is also used to describe the stability of other systems [30, 31].

Solving the replicator dynamic equations $F(x)=0$, $F(y)=0$, and $F(z)=0$, the pure strategic equilibrium points of the system are as follows: $(0,0,0),(1,0,0),(0,1,0)$, $(0,0,1),(1,1,0),(1,0,1),(0,1,1)$, and $(1,1,1)$. The Jacobian matrix of the equilibrium point $(0,0,0)$ is $J=\left[\begin{array}{lll}R_{p}-C_{\mathrm{ph}}+C_{\mathrm{pl}} & & \\ & W_{\mathrm{sb}}-C_{s}-\Upsilon_{s}-\Psi_{s}-\xi F_{s} & \\ & & F_{p}-C_{g}\end{array}\right]$, 
TABLE 2: The asymptotic stability analysis of the pure strategy equilibrium points of the replicator dynamic system.

\begin{tabular}{|c|c|c|c|}
\hline Equilibrium point & Eigenvalues $\lambda_{1}, \lambda_{2}, \lambda_{3}$ & Sign or condition & Stability \\
\hline $\begin{array}{l}(0,0,0) \\
(1,0,0)\end{array}$ & $\begin{array}{c}R_{p}-C_{\mathrm{ph}}+C_{\mathrm{pl}}, W_{\mathrm{sb}}-C_{s}-\Upsilon_{s}-\Psi_{s}-\xi F_{s}, F_{p}-C_{g} \\
C_{\mathrm{ph}}-C_{\mathrm{pl}}-R_{p},-C_{s},-C_{g}\end{array}$ & $\begin{array}{l}(+, \times, \times) \\
(-,-,-)\end{array}$ & $\begin{array}{l}\text { Unstable } \\
\text { ESS }\end{array}$ \\
\hline$(0,1,0)$ & $\begin{array}{c}C_{\mathrm{pl}}-C_{\mathrm{ph}}+C_{\mathrm{pb}}-W_{p}+\xi F_{p}, C_{s}+\Upsilon_{s}-W_{\mathrm{sb}}+\Psi_{s}+\xi F_{s}, \\
\left(F_{s}+F_{p}\right)(1-\xi)+\xi\left(C_{q}+T_{q}\right)-C_{q}\end{array}$ & $(x, x,+)$ & Unstable \\
\hline $\begin{array}{l}(0,0,1) \\
(1,1,0) \\
(1,0,1)\end{array}$ & $\begin{array}{c}R_{p}-C_{\mathrm{ph}}+C_{\mathrm{pl}}+F_{p}, W_{\mathrm{sb}}-C_{s}-\Upsilon_{s}-\Psi_{s}-F_{s}, C_{g}-F_{p} \\
C_{\mathrm{ph}}-C_{\mathrm{pl}}-C_{\mathrm{pb}}+W_{p}-\xi F_{p}, C_{s},-C_{g} \\
C_{\mathrm{ph}}-C_{\mathrm{pl}}-R_{p}-F_{p},-C_{s}, C_{g}\end{array}$ & $\begin{array}{l}(+, \times, \times) \\
(\times,+,-) \\
(-,-,+)\end{array}$ & $\begin{array}{l}\text { Unstable } \\
\text { Unstable } \\
\text { Unstable }\end{array}$ \\
\hline$(0,1,1)$ & $\begin{array}{c}C_{\mathrm{pl}}-C_{\mathrm{ph}}+C_{\mathrm{pb}}-W_{p}+F_{p}, C_{s}+\Upsilon_{s}-W_{\mathrm{sb}}+\Psi_{s}+F_{s} \\
C_{q}-\left(F_{s}+F_{p}\right)(1-\xi)-\xi\left(C_{q}+T_{q}\right)\end{array}$ & Condition (1) & ESS \\
\hline$(1,1,1)$ & $C_{\mathrm{ph}}-C_{\mathrm{pl}}-C_{\mathrm{pb}}+W_{p}-F_{p}, C_{s}, C_{g}$ & $(\times,+,+)$ & Unstable \\
\hline
\end{tabular}

Note. $\times$ indicates that the symbol is undefined; if condition (1) is not satisfied, it is unstable; Condition $(1): F_{p}<C_{\mathrm{ph}}-C_{\mathrm{pl}}-C_{\mathrm{pb}}+W_{p}$ and $C_{s}+\Upsilon_{s}+\Psi_{s}+F_{s}<W_{\mathrm{sb}}$.

TABLE 3: The asymptotic stability analysis of the hybrid strategy equilibrium points of the replicator dynamic system.

\begin{tabular}{|c|c|c|}
\hline Equilibrium point & Eigenvalues & Stability \\
\hline$\left(1,\left.y^{* *}\right|_{x=1},\left.z^{* *}\right|_{x=1}\right)$ & Equilibrium point does not exist & - \\
\hline$\left(0,\left.y^{* *}\right|_{x=0},\left.z^{* *}\right|_{x=0} ^{x=1}\right)$ & Condition (3) & Uncertain \\
\hline$\left(\left.x^{*}\right|_{y=1}, 1,\left.z^{*}\right|_{y=1}\right)$ & Condition (4) & Uncertain \\
\hline$\left(\left.x^{*}\right|_{y=0} ^{y-1}, 0,\left.z^{*}\right|_{y=0} ^{y-1}\right)$ & Equilibrium point does not exist & - \\
\hline$\left(\left.x^{* *}\right|_{z=1},\left.y^{*}\right|_{z=1}, 1\right)$ & $\exists \lambda_{i}>0$ & Unstable \\
\hline$\left(\left.x^{* *}\right|_{z=0},\left.y^{*}\right|_{z=0}, 0\right)$ & $\exists \lambda_{i}>0$ & Unstable \\
\hline$\left(\left.x^{* *}\right|_{z=z^{*}},\left.y^{* *}\right|_{x=x^{* *}},\left.z^{*}\right|_{y=y^{* *}}\right)$ & $\exists \lambda_{i}>0$ & Unstable \\
\hline$\left(\left.x^{*}\right|_{y=y^{*}},\left.y^{*}\right|_{z=z^{* *}},\left.z^{* *}\right|_{x=x^{*}}\right)$ & $\exists \lambda_{i}>0$ & Unstable \\
\hline
\end{tabular}

Note. Condition (3): if $F_{p}<C_{g}$ or $\xi F_{s}<W_{\mathrm{sb}}-\Upsilon_{s}-\Psi_{s}-C_{s}$, this point exists and in a critical state. Condition (4): if $\xi F_{p}<C_{\mathrm{ph}}-C_{\mathrm{pl}}-C_{\mathrm{pb}}+W_{p}$, this point exists and in a critical state.

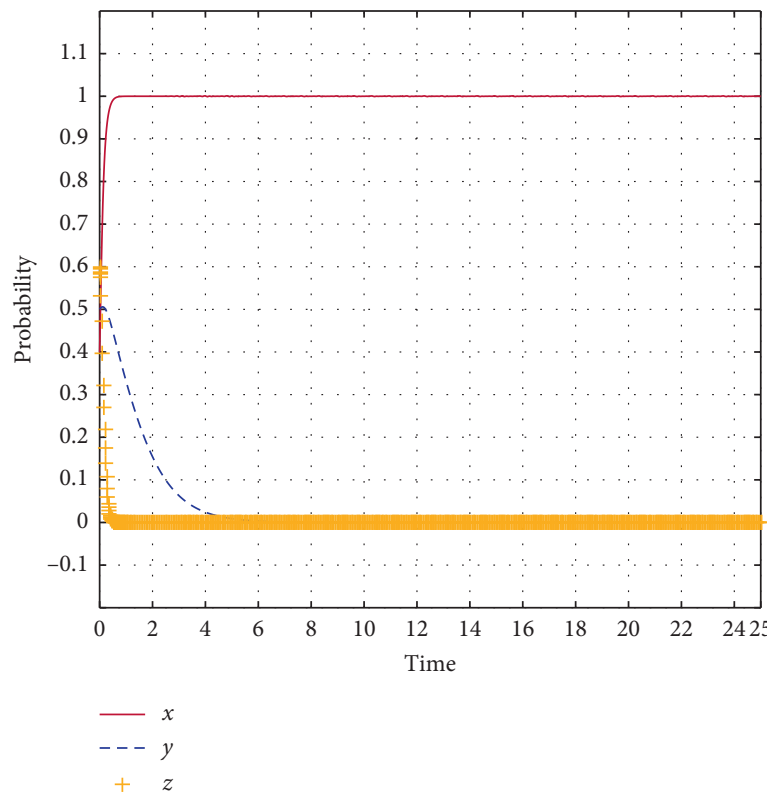

(a)

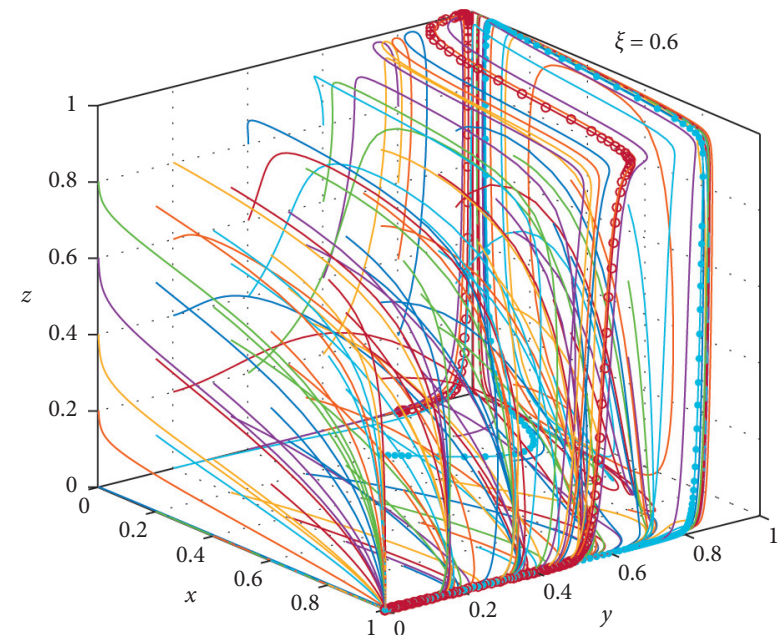

(b)

Figure 5: The evolution result of the replicator dynamic system.

and its eigenvalues are $\lambda_{1}=R_{p}-C_{\mathrm{ph}}+C_{\mathrm{pl}}, \lambda_{2}=W_{\mathrm{sb}}-C_{s}-$ $\Upsilon_{s}-\Psi_{s}-\xi F_{s}$, and $\lambda_{3}=F_{p}-C_{g}$. Because $\lambda_{1}>0$, its eigenvalues have at least one positive number, and $(0,0,0)$ is unstable. Similarly, the asymptotic stability analysis of the replicator dynamic system's pure dynamic equilibrium points is shown in Table 2.
From Table 2, there is at least one stable strategy combination in the pure dynamic equilibrium points (i.e., pharmaceutical companies cleaner producing high-quality drugs, medical service agencies' noncollusion, and the governments choosing loose regulation). In addition, if the government's fines for pharmaceutical companies are not 


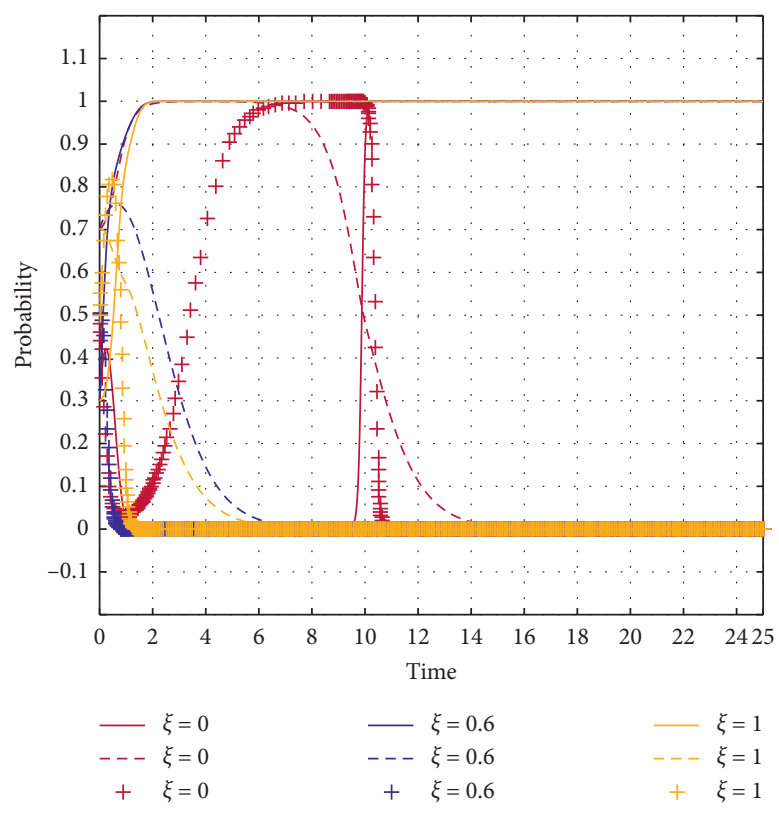

(a)

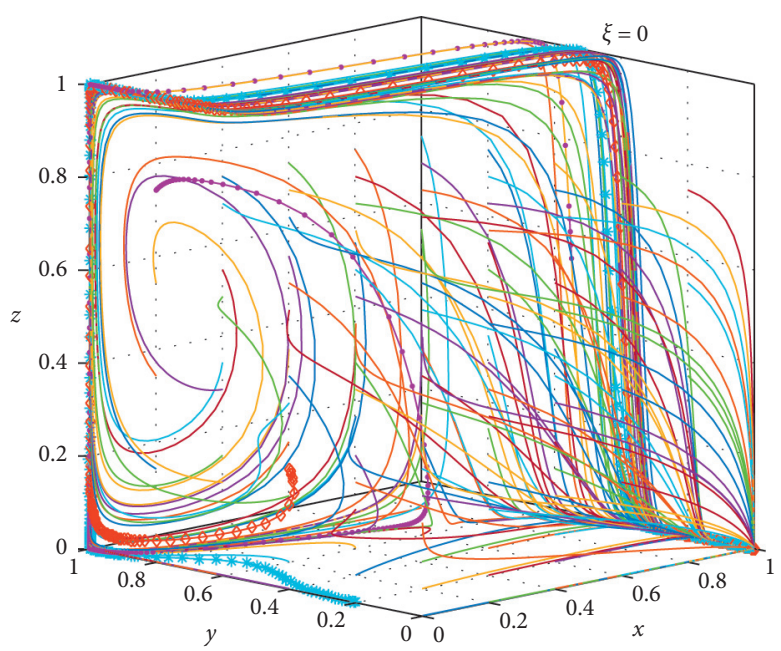

(b)

FIgURE 6: The impact of patient complaint rates.

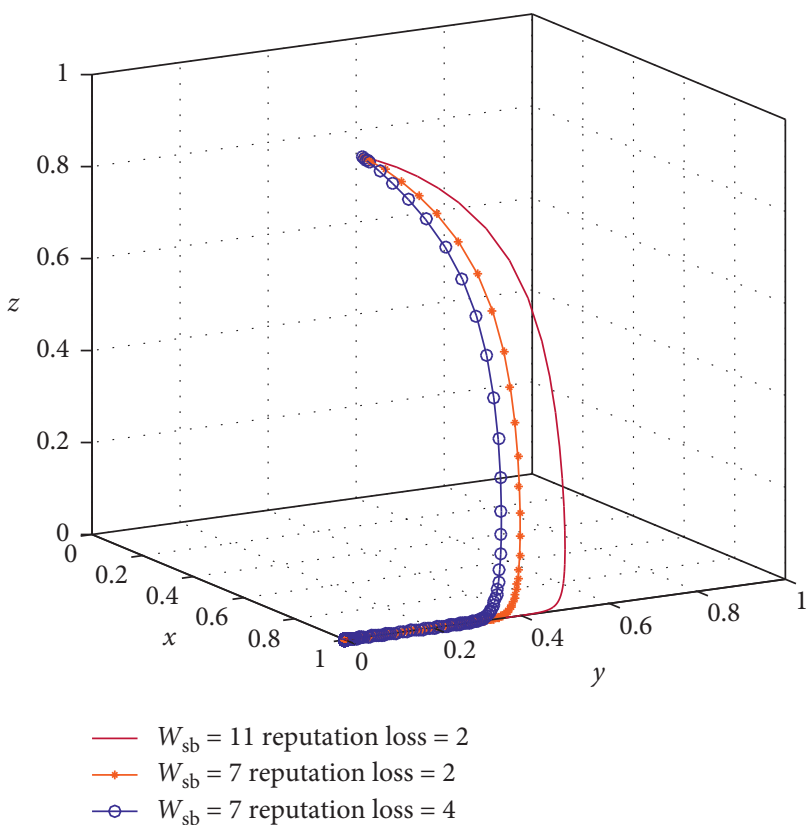

Figure 7: The impact of collusion income and reputation loss.

large enough (less than a certain threshold) and the conspiracy benefits of medical service agencies are high, the replicator dynamic system will have another combination of stability strategies (i.e., drug companies providing lowquality drugs, medical service agencies choosing collusion, and the government choosing strict regulation). To avoid collusion, the governments have to investigate drug companies' speculative income comprehensively, strengthen penalties, and create a zero-tolerance regulatory mechanism for drug quality issues.

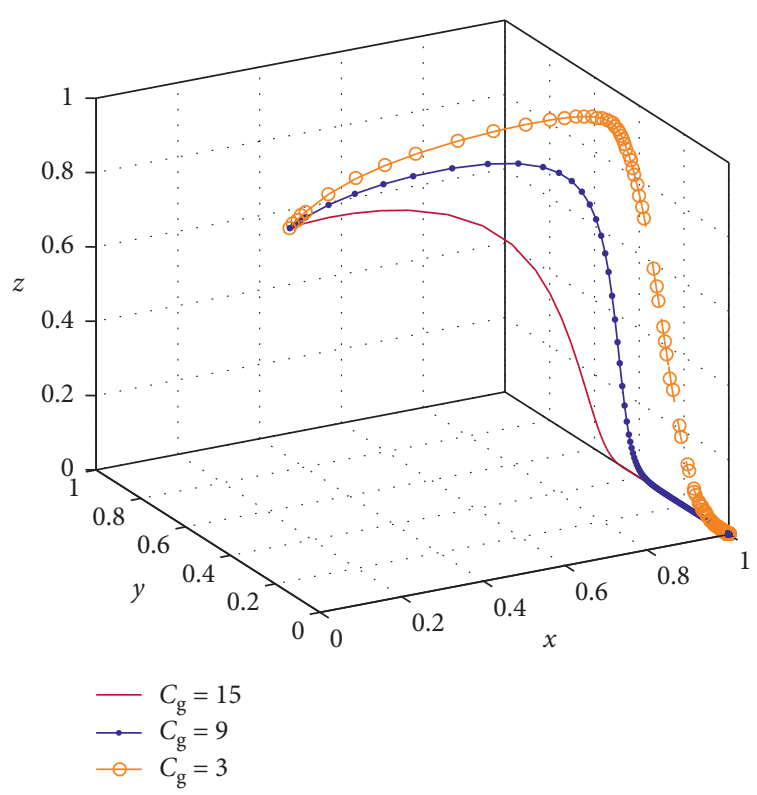

FIGURE 8: The impact of the cost of strict regulation.

From the equations $F(x)=0, F(y)=0$, and $F(z)=0$, there are at most eight hybrid strategic equilibrium points for the replicator dynamic system. In addition, every strategy has a value range of $[0,1]$; otherwise, the point does not exist. Similarly, the asymptotic stability analysis of the hybrid strategy equilibrium points is shown in Table 3 . There are two possible hybrid strategic equilibrium points, and the stability of these two points cannot be determined by the eigenvalues; we judged the stability point by numerical simulation in Section 5. 

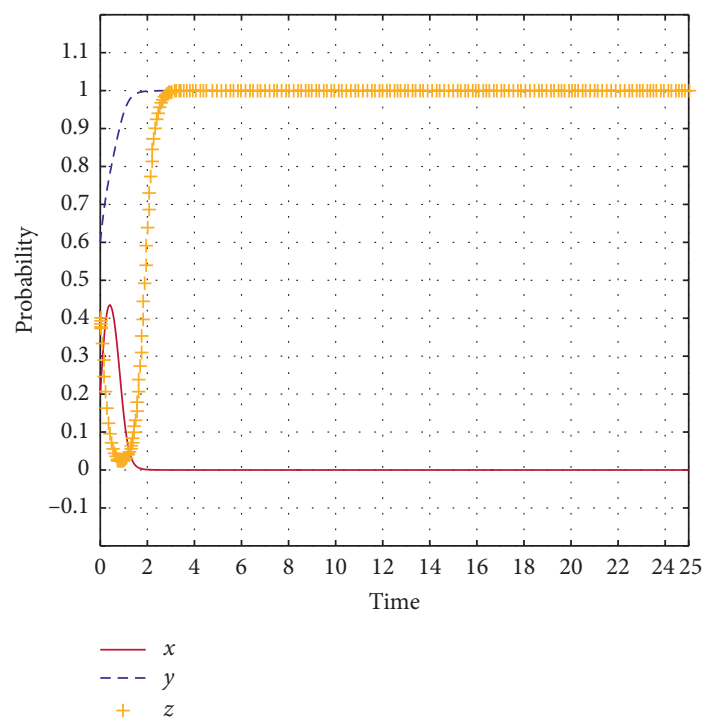

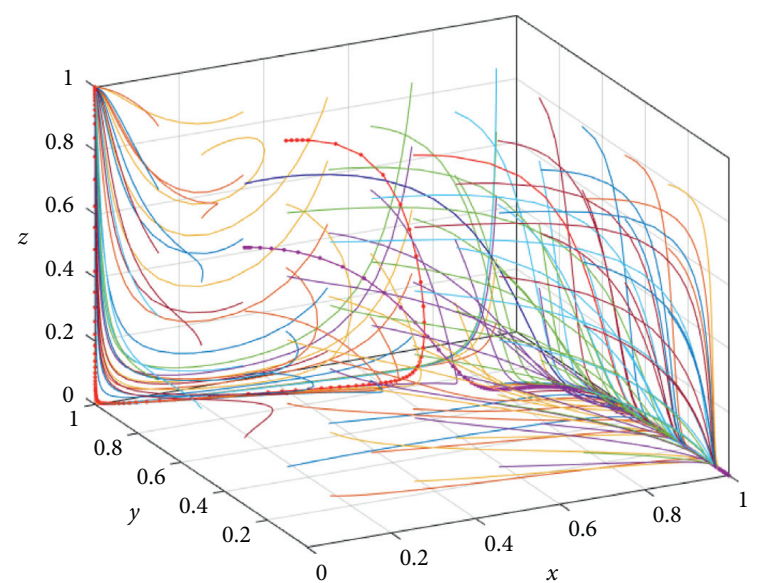

(b)

(a)

FIGURE 9: The impact of reducing fines for pharmaceutical companies.

As can be seen in Table 3, according to the conditions under which the two points with uncertain stability exist, if the government regulation authorities reduced the cost of strict regulation and increased the fines for illegal drug companies and illegal medical service agencies, the two equilibrium points with uncertain stability will be nonexistent. This is a way to completely eliminate the possibility of low-quality drugs flowing to patients. In addition, improving the patients' medical knowledge and awareness of their rights by extensive public education is also an effective measure to ensure the healthy development of high-quality cleaner production in the pharmaceutical industry.

\section{Simulation Analysis}

To further verify the stability of the evolutionary game equilibrium points in the game system and the relationship of each element, we used the Matlab 2017b software to perform simulation analysis by assigning values to related elements under different conditions.

When the fine for the pharmaceutical companies is greater than the companies' speculative income but is less than their speculative gains in the case of loose regulation, assuming the probability of a patient complaint is $\xi=0.6$, then the values of the other elements are as follows: $R_{p}=30$, $C_{\mathrm{ph}}-C_{\mathrm{pl}}=10, C_{\mathrm{pb}}=7, W_{p}=8, F_{p}=12, W_{\mathrm{sb}}=11, \Psi_{s}=2$, $\Upsilon_{s}=1, C_{s}=1, F_{s}=4.5, C_{g}=15$, and $T_{g}=20$. The results of the dynamic evolution starting from the initial point $(0.4,0.5,0.6)$ and from different initial points are shown in Figure 5 . There is only one asymptotic stability point $(1,0,0)$ for the replicator dynamic systems.

As can be seen in Figure 5, pharmaceutical companies steadily cleaner produce high-quality drugs, medical service agencies choose noncollusion, and the drug market can selfregulate and maintain healthy development. At this time, the pressure of government regulation has been reduced, so that loose regulation can be adopted. It can be seen that two equilibrium points with unstable stability are unstable. Moreover, the evolution of pharmaceutical companies to cleaner produce high-quality drugs is faster than the evolution of medical service agencies to choose noncollusion.

With the patients' complaint rate increases from 0 to 0.6 and then increases to 1 , the results of the dynamic evolution starting from the initial point $(0.4,0.5,0.6)$ and from different initial points are shown in Figure 6.

Obviously, with the increasing complaint rate of patients, both cleaner production pharmaceutical companies and medical service agencies will evolve to provide highquality drugs and adopt noncollusion at a faster rate. When patients' complaint rate is 0 , the final game system will evolve to a stable strategy combination point in which the pharmaceutical companies cleaner produce high-quality drugs, the medical service agencies choose noncollusion, and the governments choose loose regulation. But, the speed of evolution to the stable point is slowed, when the probability of collusion by medical service agencies as an initial strategy is high. And, there are cases in the process of evolution in which pharmaceutical companies provide low-quality drugs and medical service agencies choose collusion. With the government regulatory authorities implementing stricter regulation, low-quality drugs will gradually be expelled from the market, which will make the drug market adjust to a healthy and stable development model after a long period of time.

Under a condition in which other numerical conditions are unchanged, the change in income of the medical service agencies gained from conspiracy from 11 to 7 and the change in reputation loss from 2 to 4 were analyzed, and the process and results of the dynamic evolution from the initial strategy combination $(0.2,0.5,0.8)$ are shown in Figure 7.

From the evolution process, when the conspiracy benefits of the medical service agencies reduced and the 
reputation loss of medical service agencies increased, the medical service agencies can evolve to noncollusion faster.

As the costs of governments' strict regulatory are reduced from 15 to 9 and continue to decrease to 3, the results of the dynamic evolution from the initial strategy portfolio $(0.4,0.6,0.5)$ are shown in Figure 8.

From the evolutionary process, it can be seen that reducing the cost of strict regulation by the governments can improve the rate of strict regulation in the evolutionary process; in this situation, the speed of evolution from strict regulation to loose regulation slows down, after the pharmaceutical companies steadily cleaner produce high-quality drugs. Therefore, the governments can more effectively resist the fluctuation of the drug market and stabilize the highquality development of cleaner production in the pharmaceutical industry by innovating low-cost regulation.

Finally, on the basis of the original value, reducing the fines of the pharmaceutical companies to 8 will not effectively curb the speculative behavior of pharmaceutical companies and medical service agencies. The evolution results are shown in Figure 9.

There are two gradual stabilization points $(1,0,0)$ and $(0,1,1)$. In this situation, there are some low-quality drugs in the market that endanger the lives and health of patients. Therefore, it is important for the government to increase fines for such drugs. If the fines are too weak, the governments cannot effectively restrict collusion between pharmaceutical companies and medical service agencies, which will certainly bring harm to public health; if the fines are strong, the government can eliminate the speculative phenomenon and create a good atmosphere for the cleaner production of high-quality medicines.

\section{Conclusions}

Improving the regulation of cleaner production quality and ensuring the safety of drug use by the public are urgent tasks for all countries in the world. To avoid the conspiracy between medical service agencies and pharmaceutical companies to produce low-quality drugs, we constructed a tripartite evolution game model and analyzed the conditions needed for cleaner production pharmaceutical companies to stably provide high-quality drugs. We obtained a variety of measures to promote the quality of drugs, including the impact of patient feedback and other factors. The main conclusions are as follows.

Firstly, the improvement of patient complaint rates can effectively curb collusive behavior and strengthen the stability of cleaner production of high-quality medicines; therefore, the governments should attach importance to public education and improve the public's medical knowledge and awareness of safeguarding their rights by publishing brochures and recording promotional films. Secondly, the fines for pharmaceutical companies must be larger than a certain threshold to avoid a gradual stabilization point under collusive behavior; therefore, the government must punish the violation of laws and regulations with the severest penalties and set a zero-tolerance regulatory policy for inferior pharmaceuticals. Thirdly, enhancing the patient feedback mechanism is conducive to accelerating the evolution of pharmaceutical companies and medical service agencies to choose legitimate strategies. Thus, the necessity of a web-based evaluation system and regular drug quality-based activities should not be neglected. Finally, it is important for the governments to reduce the cost of strict regulation using innovative methods; with lower regulatory costs, the government can evolve to loose regulation at a slower pace and the stability of pharmaceutical companies cleaner producing high-quality drugs can be improved.

Pharmaceutical quality regulation is a complex problem affected by multiple parties. This paper only considers the stage in which cleaner production pharmaceutical companies are providing drugs to patients through medical service agencies, ignoring the impact of other companies at all levels and ignoring the impact of the game sequence. Therefore, we will further study the cooperation and constraint relationships among distributors, retailers, and the government under incomplete information and the dynamic game model and further research pharmaceutical regulation strategies for the multichannel drug supply chain in an Internet-based environment.

\section{Data Availability}

All the data are available within the manuscript.

\section{Conflicts of Interest}

The authors declare that no conflicts of interest exist.

\section{Acknowledgments}

This work was supported by the Humanities and Social Sciences Foundation of the Ministry of Education in China under grant no. 17YJA630147, Nature Science Foundation of Shandong Province under grant no. ZR2019MG017, and National Social Science Foundation of China under grant no. 13AGL012.

\section{References}

[1] B. Yavuztürk, S. Bucak, and M. Özilgen, "Waste generation, product yield evaluation and exergy analysis during bisphosphonate synthesis and medical drug production processes," Journal of Cleaner Production, vol. 198, pp. 242257, 2018.

[2] Z. Gao, Y. Geng, R. Wu, W. Chen, F. Wu, and X. Tian, "Analysis of energy-related $\mathrm{CO}_{2}$ emissions in China's pharmaceutical industry and its driving forces," Journal of Cleaner Production, vol. 223, pp. 94-108, 2019.

[3] L. Chan and T. Daim, "A research and development decision model for pharmaceutical industry: case of China," R\&D Management, vol. 48, no. 2, pp. 223-242, 2017.

[4] Q. Sun, M. A. Santoro, Q. Meng, C. Liu, and K. Eggleston, "Pharmaceutical policy in China," Health Affairs, vol. 27, no. 4, pp. 1042-1050, 2008.

[5] L. L. Yan, X. Yan, Y. Tan, and S. X. Sun, "Shared minds: how patients use collaborative information sharing via social media platforms," Production and Operations Management, vol. 28, no. 1, pp. 9-26, 2019. 
[6] A. Albekairy and A. M. Abdulkareem, "Does the patients' educational level and previous counseling affect their medication knowledge?" Annals of Thoracic Medicine, vol. 8, no. 2, pp. 105-108, 2013.

[7] T. M. Alshammari, "Drug safety: the concept, inception and its importance in patients' health," Saudi Pharmaceutical Journal, vol. 24, no. 4, pp. 405-412, 2016.

[8] B. R. Gibson, R. Suh, and H. Tilson, "The US drug safety system: role of the pharmaceutical industry," Pharmacoepidemiology and Drug Safety, vol. 17, no. 2, pp. 110-114, 2008.

[9] X. Li and D. Hamblin, "Factors impacting on cleaner production: case studies of Chinese pharmaceutical manufacturers in Tianjin, China," Journal of Cleaner Production, vol. 131, pp. 121-132, 2016.

[10] A. Marucheck, N. Greis, C. Mena, and L. Cai, "Product safety and security in the global supply chain: issues, challenges and research opportunities," Journal of Operations Management, vol. 29, no. 7-8, pp. 707-720, 2011.

[11] J. S. Busby, "The co-evolution of competition and parasitism in the resource-based view: a risk model of product counterfeiting," European Journal of Operational Research, vol. 276, no. 1, pp. 300-313, 2019.

[12] G. Bardwell, J. Boyd, J. Arredondo, R. McNeil, and T. Kerr, "Trusting the source: the potential role of drug dealers in reducing drug-related harms via drug checking," Drug and Alcohol Dependence, vol. 198, pp. 1-6, 2019.

[13] M. M. Parast and D. Golmohammadi, "Quality management in healthcare organizations: empirical evidence from the baldrige data," International Journal of Production Economics, vol. 216, pp. 133-144, 2019.

[14] E. G. Campbell, R. L. Gruen, J. Mountford et al., "A national survey of physician-industry relationships," New England Journal of Medicine, vol. 357, no. 5, pp. 507-508, 2007.

[15] E. Yung and S. Stremersch, "Drug detailing and doctors' prescription decisions: the role of information content in the face of competitive entry," Marketing Science, vol. 35, no. 6, pp. 915-933, 2016.

[16] L. J. Paulozzi, “Prescription drug overdoses: a review," Journal of Safety Research, vol. 43, no. 4, pp. 283-289, 2012.

[17] J. Tang, R. Shao, F. J. Lin, and X. Gao, "Evaluation of adverse drug reaction (ADR) monitoring and reporting system in China," Value in Health, vol. 17, no. 7, p. A791, 2014.

[18] R. Donelan, S. Walker, and S. Salek, "Factors influencing quality decision-making: regulatory and pharmaceutical industry perspectives," Pharmacoepidemiology and Drug Safety, vol. 24, no. 3, pp. 319-328, 2015.

[19] S. M. Blass and J. V. Gray, "Inter-organizational quality management: the use of contractual incentives and monitoring mechanisms with outsourced manufacturing," Production and Operations Management, vol. 22, no. 6, pp. 1540-1556, 2013.

[20] H. Yi, G. Miller, L. Zhang, S. Li, and S. Rozelle, "Intended and unintended consequences of China's zero markup drug policy," Health Affairs, vol. 34, no. 8, pp. 1391-1398, 2015.

[21] W. Liu, Z. Wan, Z. Wan, and B. Gong, "Sustainable recycle network of heterogeneous pharmaceuticals with governmental subsidies and service-levels of third-party logistics by bi-level programming approach," Journal of Cleaner Production, vol. 249, Article ID 119324, 2020.

[22] W. Du, J. J. Guo, Y. Jing, X. Li, and C. M. L. Kelton, "Drug safety surveillance in China and other countries: a review and comparison," Value in Health, vol. 11, pp. s130-s136, 2008.

[23] W. He, D. Yao, Y. Hu, and H. Dai, "Analysis of a pharmacist-led adverse drug event management model for pharmacovigilance in an academic medical center hospital in China," Therapeutics and Clinical Risk Management, vol. 14, pp. 2139-2147, 2018.

[24] S. Encarnação, F. P. Santos, F. C. Santos, V. Blass, J. M. Pacheco, and J. Portugali, "Paths to the adoption of electric vehicles: an evolutionary game theoretical approach," Transportation Research Part B: Methodological, vol. 113, pp. 24-33, 2018.

[25] W. C. Abram and K. Noray, "Political corruption and public activism: an evolutionary game-theoretic analysis," Dynamic Games and Applications, vol. 8, no. 1, pp. 1-21, 2017.

[26] Z.-Z. Jiang, N. He, X. Qin, W. H. Ip, C. H. Wu, and K. L. Yung, "Evolutionary game analysis and regulatory strategies for online group-buying based on system dynamics," Enterprise Information Systems, vol. 12, no. 6, pp. 695-713, 2018.

[27] J. Luo, J. Wang, Y. Zhao, and T. Chen, "Evolution model of health food safety risk based on prospect theory," Journal of Healthcare EngineeringComplexity, vol. 2018, Article ID 8769563, 12 pages, 2018.

[28] R. Long, J. Yang, H. Chen, Q. Li, W. Fang, and L. Wang, "Coevolutionary simulation study of multiple stakeholders in the take-out waste recycling industry chain," Journal of Environmental Management, vol. 231, pp. 701-713, 2019.

[29] Q. Liu, X. Li, and M. Hassall, "Evolutionary game analysis and stability control scenarios of coal mine safety inspection system in China based on system dynamics," Safety Science, vol. 80, pp. 13-22, 2015.

[30] Z. Lu, P. Huang, and Z. Liu, "Predictive approach for sensorless bimanual teleoperation under random time delays with adaptive fuzzy control," IEEE Transactions on Industrial Electronics, vol. 65, no. 3, pp. 2439-2448, 2018.

[31] A. G. Reidemeister and S. I. Levytska, "Stability of motion of railway vehicles described with lagranged equations of the first kind," Science and Transport Progress. Bulletin of Dnipropetrovsk National University of Railway Transport, vol. 77, no. 5(77), pp. 93-103, 2018. 